\title{
Performing precision measurements and new physics searches at the HL-LHC with the upgraded CMS Level-1 Trigger
}

\section{Emily MacDonald ${ }^{\dagger * *}$}

University of Colorado Boulder, Boulder, Colorado, USA

E-mail: emily.macdonald@colorado.edu

The High-Luminosity LHC will open an unprecedented window on the weak-scale nature of the universe, providing high-precision measurements of the standard model as well as searches for new physics beyond the standard model. Such precision measurements and searches require information-rich datasets with a statistical power that matches the high luminosity provided by the Phase-2 upgrade of the LHC. Efficiently collecting those datasets will be a challenging task, given the harsh environment of 200 proton-proton interactions per LHC bunch crossing. For this purpose, CMS is designing an efficient data-processing hardware trigger (Level-1) that will include tracking information and high-granularity calorimeter information. The current conceptual system design is expected to take full advantage of FPGA and link technologies over the coming years, providing a high-performance, low-latency computing platform for large throughput and sophisticated data correlation across diverse sources. The envisaged L1 system will more closely replicate the full offline object reconstruction to perform a more sophisticated and optimized selection. Algorithms such as particle flow reconstruction can be implemented and complemented by standalone trigger object reconstruction. The expected performance and physics implications of such algorithms are studied using Monte Carlo samples with high pile-up, simulating the harsh conditions of the HL-LHC. The trigger object requirements are not only driven by the need to maintain physics selection thresholds to match those of the Phase-1, the selection of exotic signatures including displaced objects must be provided to help expand the physics reach of the experiment. The expected acceptance increase on selected benchmark signals obtained by the upgraded CMS Phase-2 Level-1 trigger will be summarized in this paper.

40th International Conference on High Energy physics - ICHEP2020

July 28 - August 6, 2020

Prague, Czech Republic (virtual meeting)

\footnotetext{
$\dagger$ on behalf of the CMS collaboration

*Speaker
} 


\section{Introduction}

The CMS Phase-2 physics program will fully exploit the High Luminosity configuration (HLLHC), with an integrated luminosity of $4000 \mathrm{fb}^{-1}$ and an increased average number of proton-proton collisions per bunch crossing (pileup) of around 200. CMS has implemented a two-level triggering system composed of the Level-1 (L1) trigger, which operates at the hardware level, and a software High Level Trigger (HLT). The L1 trigger relies on the implementation of higher granularity detectors, using the information of the calorimeters, the extended- $\eta$ range of the muon chambers, and now the tracker, to drastically decrease the rate from $40 \mathrm{MHz}$ to an output of $750 \mathrm{kHz}$. The upgrade of the trigger system will enhance the physics selectivity and maintain the performance necessary throughout the 10 year-long HL-LHC program.

\section{Upgrades to the L1 Trigger}

The Phase-2 L1 trigger upgrades consist of improvements to both hardware and algorithms. The upgraded hardware components include new FPGAs (Xilinx UltraScale+ class) and improved high-speed optical links, up to $28 \mathrm{~Gb} / \mathrm{s}$. The total latency for the trigger has been increased to 12.5 $\mu \mathrm{s}$, which enables the use of tracker and high-granularity calorimeter information, and also the potential to use particle-flow techniques [2] or a greater use of machine-learning based approaches.

The trigger consists of four independent data processing paths, and a final global trigger that receives all output objects and issues the final L1 trigger decision. The calorimeter trigger builds electron candidates, hadronically decaying taus, jets, and associated energy sums. The muon trigger generates track-matched muons, with an extended coverage now up to $|\eta|=2.8$. The track trigger reconstructs tracks (not available in Phase-1), primary vertices, and tracker-only based objects, such as jets and energy sums. Lastly, the correlator trigger produces a global event reconstruction. It creates particle-flow candidates and hosts a version of the Pileup Per Particle Identification (PUPPI) algorithm [3], which removes pileup.

\section{Physics Reach}

A broad spectrum of physics analyses will become possible with the new capabilities offered by the detector upgrade, the inclusion of tracking at Level-1, and the use of particle-flow algorithms. Many of the upgraded trigger algorithms achieve lower rates, even at 200 pileup, as can be seen in Figure 1. This releases some bandwidth. We can then reduce some thresholds in order to increase the acceptance for key physics signals, such as channels that trigger using leptons or PUPPI $E_{T}^{m i s s}$.

\subsection{Displaced jets, machine learning, and particle-flow}

An extension of the L1 track-finding, clustering tracks without a beamspot constraint into jets provides a new handle to trigger on Beyond the Standard Model (BSM) physics. The improved L1 performance with the displaced jets can be seen in Figure 2 .

Using particle-flow reconstructed jets allows the thresholds used by the Phase-1 L1 trigger to remain similar for HL-LHC. In some cases, such as for the dedicated VBF trigger algorithm, the signal acceptance can be significantly improved, as can be seen in Figure 2. 

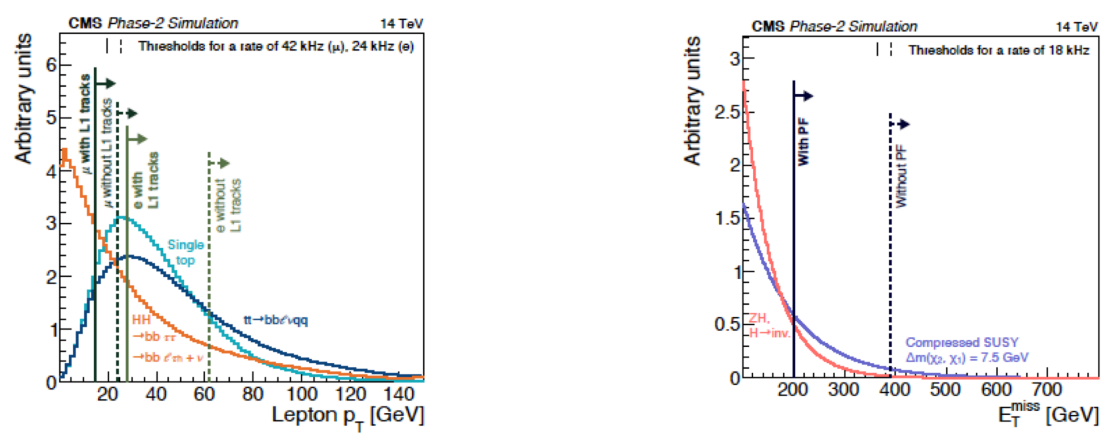

Figure 1: Threshold reductions for lepton $\mathrm{p}_{\mathrm{T}}$ (left) and PUPPI $\mathrm{E}_{T}^{\text {miss }}$ (right). The use of tracking substantially decreases the lepton $\mathrm{p}_{\mathrm{T}}$ threshold, from $62 \mathrm{GeV}$ to $28 \mathrm{GeV}$ for electrons, and from $25 \mathrm{GeV}$ to $15 \mathrm{GeV}$ for muons. Particle-flow techniques reduce the $\mathrm{E}_{T}^{\text {miss }}$ threshold from $390 \mathrm{GeV}$ to $200 \mathrm{GeV}$.
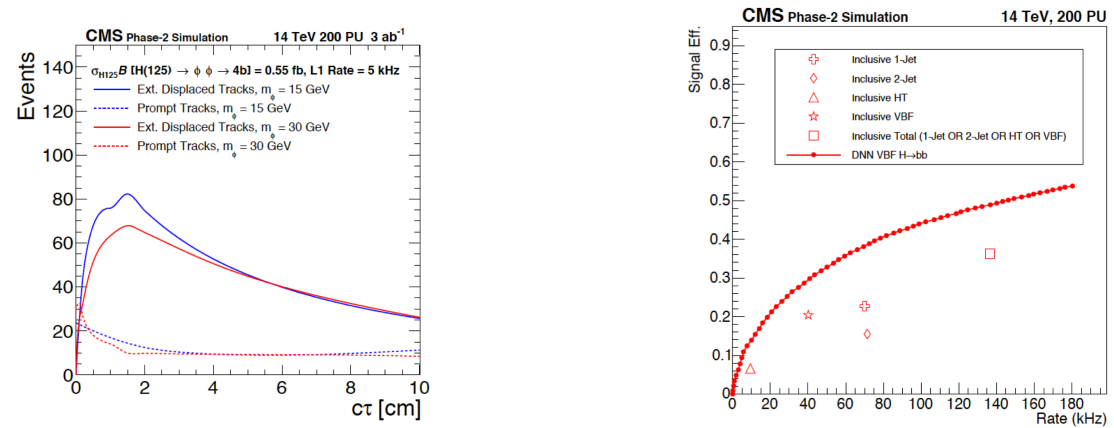

Figure 2: The improved L1 performance using displaced jets can be seen in the almost $6 \mathrm{x}$ increased acceptance (left) for the SM Higgs boson. The signal acceptance for an inclusive VBF $\mathrm{H} \rightarrow \mathrm{b} \overline{\mathrm{b}}$ trigger (right) can be significantly improved using particle-flow reconstructed jets. Use of a deep neural net outperforms all cut-based algorithms, with an efficiency of 0.49 compared to 0.36 .

\subsection{Scouting system}

Trigger scouting harvests physics objects produced at various levels of the trigger system, and only stores high-level information. This system has the advantage of systematically searching for correlations among sequential bunch crossings, and can be used to identify potential signatures unreachable through standard trigger processes. The scouting system will provide real-time trigger component diagnostics and could benefit a variety of physics channels, such as: rare Higgs decays, single- $\tau$ final states, and QCD measurements with high statistics.

\section{References}

[1] CMS Collaboration, The Phase-2 Upgrade of the CMS Level-1 Trigger, CERN-LHCC-2020001, CMS-TDR-20-001 (2020)

[2] Particle-flow reconstruction and global event description with the CMS detector, CMS, JINST 12 P10003 (2017).

[3] Pileup Per Particle Identification, D. Bertolini, P. Harris, M. Low, and N. Tran, JHEP 10 (2014) 059. 